environments on most continents have grown bluer with climate change, so animal populations may also become bluer.

Previous models have suggested that those red populations that shift towards blue are less prone to extinction, although other factors, such as habitat destruction, could counteract any benefit.

J. Anim. Ecol. doi:10.1111/j.1365 2656.2011.01833.x (2011)

\section{ANIMAL BEHAVIOUR}

\section{Birds with beauty} - and brains

Some birds have it all. Male siskins with brighter, more attractive plumage are better at finding food than their less eye-catching counterparts.

Fernando Mateos-Gonzalez and his team at the Natural History Museum of Barcelona, Spain, measured the length of a yellow stripe on the wings of male siskins (Carduelis spinus; pictured). Females are known to pick mates on the basis of the length of this stripe. The researchers challenged hungry males to unlock a cache of pine seeds blocked by toothpicks.

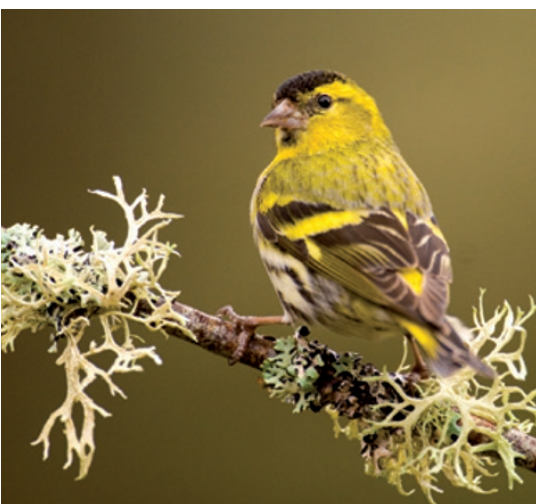

Birds with longer yellow stripes solved the problem more quickly than those with shorter stripes. The authors ruled out age, size and social dominance as possible contributory factors, and conclude that fancier feathers might help discerning females to select mates that are not only sexier, but also smarter. Biol. Lett. doi:10.1098/ rsbl.2011.0163 (2011)

\section{Saturated fats up inflammation}

A diet high in saturated fats raises the risk of type 2 diabetes - perhaps owing to the activity of an inflammatory protein complex called the inflammasome.

Jenny Ting and her team at the University of North Carolina at Chapel Hill found that a saturated fatty acid called palmitate stimulates the inflammasome - which activates the inflammatory response - in cultured mouse macrophages, a type of immune cell. An unsaturated fat did not do this. The macrophages, in turn, produced a potent inflammatory molecule called IL- $1 \beta$ that blocked insulin signalling in cultured liver cells. Macrophages lacking key components of the inflammasome did not interfere with insulin signalling.

Mice fed a high-fat diet for 12 weeks developed insulin resistance in liver, fat and muscle tissue, along with other signs of type 2 diabetes, but those that lacked inflammasome genes did not. Nature Immunol. doi:10.1038/ ni.2022 (2011) \section{CANCER \\ Stem-cell genes linked to relapse}

The recurrence of colon cancer in patients after treatment is linked to a pattern of genes expressed in the tumour that is specific to intestinal stem cells.

Eduard Batlle at the Institute for Research in Biomedicine in Barcelona, Spain, and his colleagues isolated cells from pockets in the mouse intestinal wall known to house stem cells. They found that intestinal stem cells had the highest expression of a gene called Ephb2, and that about 50 other genes were also upregulated. Analysis of samples from 340 patients with colon cancer revealed an association between high

COMMUNITY CHOICE

The most viewed papers in science

\title{
Laser from a tiny wire
}

\section{HIGHLY READ \\ on pubs.acs.org in March}

Lasers made from nanometre-scale wires could be valuable for small, fast, opticalcommunication and signal-processing devices, but researchers have been unable

to tune the wavelength of the light emitted.

Limin Tong at Zhejiang University in Hangzhou, China, and his colleagues have made a laser from a cadmium selenide wire 200 nanometres in diameter and bent into two loops resembling an elongated figure of eight. The loops act as mirrors to reflect the light back and forth, boosting the light's intensity. When the researchers decreased the size of one of the loops, the peak wavelength of the light shifted by 7 nanometres, suggesting that lasers created in this way could be made fully tunable.

Nano Lett. 11, 1122-1126 (2011) expression levels of these genes and increased risk of relapse.

When human tumour cells were implanted in mice, cells expressing the highest levels of Ephb2 showed the greatest capacity for initiating tumours

Cell Stem Cell doi:10.1016/

j.stem.2011.02.020 (2011)

\section{CLIMATE CHANGE}

\section{Cold spells in a warm world}

Severe cold streaks could persist well into the future despite global warming, a climate modelling study finds.

Auroop Ganguly and his colleagues at Oak Ridge National Laboratory in Tennessee compared data from extreme cold events between 1991 and 2000 with projections made by nine global climate models for 2091-2100. Their results show that by the end of this century, extreme cold snaps in many parts of the world could be more intense and more prolonged although less frequent - than the twentieth-century average.

The team suggests that governments will still need to plan for cold spells even as the world warms.

Geophys. Res. Lett. doi:10.1029/ 2011GL047103 (2011) at low cell dosages.

\section{MOLECULAR BIOLOGY} RNA goes solo

Researchers have engineered an RNA enzyme to synthesize another active RNA enzyme from an RNA template. This models one theory for how life originated on Earth: with RNA molecules that both encoded genetic information and catalysed reactions to express that information.

Philipp Holliger and his co-workers at the Medical Research Council Laboratory of Molecular Biology in Cambridge, UK, started out with an RNA enzyme, or ribozyme, called R18, which is known to synthesize short stretches of RNA. They created a library of modified versions of R18 and used it to screen for ribozymes with improved enzymatic activity. They ended up with a new ribozyme, tC19Z, capable of generating longer pieces of RNA from a wider range of templates than R18. The enzyme could also synthesize a different ribozyme.

Science 332, 209-212 (2011)

\section{ONATURE.COM}

For the latest research published by Naturevisit:

www.nature.com/latestresearch

\section{No DNA needed,}

